\title{
Countdown zur Fortbildung im Schnee läuft
}

„Gemeinsam hoch hinaus" lautet das Motto des Winterkongresses des Der Freien Verbandes Deutscher Zahnärzte (FVDZ) im österreichischen Ischgl. Vom 28. Februar bis 4. März 2016 erwartet Zahnärzte und Praxismitarbeiter ein abwechslungsreiches Kongressprogramm. International renommierte Referenten stellen neue Therapiekonzepte und Diagnoseverfahren vor.

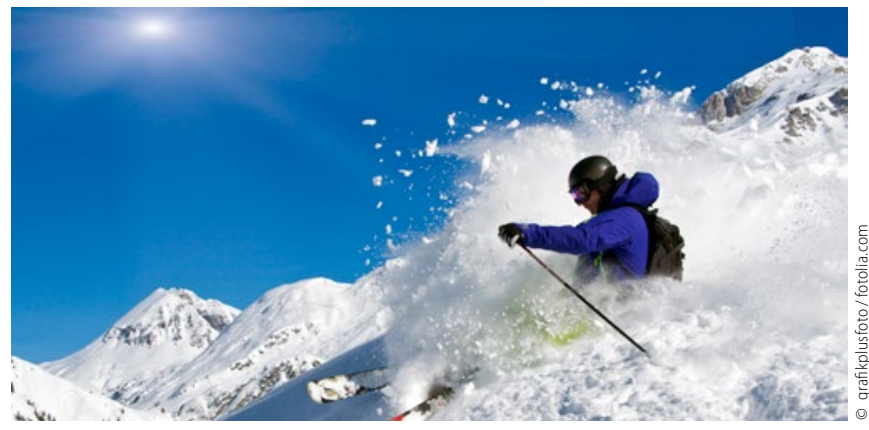

Themen wie Neuroplastizität in der Kieferorthopädie, Zahntransplantation und Herausforderungen bei implantatprothetischen Versorgungen stehen auf dem Programm. Im berufs- und standespolitischen Teil des Kongresses informiert der Geschäftsführende Bundesvorstand über die Reform der Gebührenordnung für Ärzte, die ebenfalls Berechnungsgrundlage für einen Teil der zahnärztlichen Leistungen ist, sowie über deren Bedeutung für eine eventuelle Novelle der Gebührenordnung für Zahnärzte.

Ein weiterer Programmhöhepunkt: Bei der Eröffnungsveranstaltung stellt der Musiker, Unternehmer und Ausdauersportler Joey Kelly in dem Vortrag „No Limits - Wie schaffe ich mein Ziel“ seine Motivationsstrategien vor, die ihn zu zahlreichen sportlichen Erfolgen führten. Das detaillierte Programm kann unter $w w w . f v d z$.de eingesehen oder bei der Bundesgeschäftsstelle angefordert werden. Ansprechpartnerin dort ist Alisa Pickel unter der Telefonnummer 0228 - 855751 oder per Mail unter kongresse@fvdz.de.

\section{Zahnärztlicher Austauschdienst}

\section{Birgit Hauer ist neue ZAD-Geschäftsführerin}

Am Rande der diesjährigen Bundesfachschaftstagung Zahnmedizin in Hamburg ist die Studentenbeauftragte des Freien Verbandes Deutscher Zahnärzte (FVDZ), Birgit Hauer, zur Geschäftsführerin des Zahnärztlichen Austauschdienstes (ZAD)

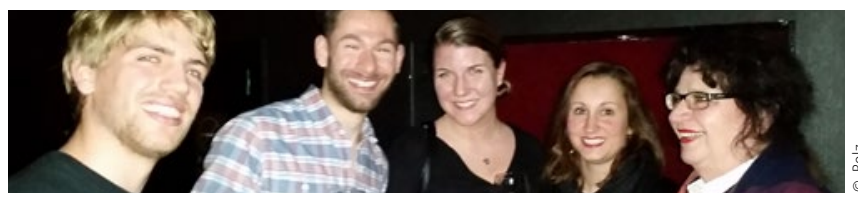

Nach der Wahl zur ZAD-Geschäftsführerin: Birgit Hauer (2. v.re.) mit ZAD-Vorstand Jonas Kilger, Christoph Beckmann, Alma Wiedenhofer und Assistentin der ZAD-Geschäftsführung Doris Bungartz (von links nach rechts) gewählt worden. Der Freie Verband ist seit fast 35 Jahren eng mit dem ZAD verbunden und stellt personelle Unterstützung zur Verfügung. Ideen für gemeinsame Projekte hatten der Vorstand des ZAD und die neue Geschäftsführerin bereits bei ihrem ersten Treffen im Dezember gesammelt - nun geht es an die Umsetzung. Der junge Vorstand kümmert sich gemeinsam mit Hauer darum, die Außendarstellung des ZAD zu überarbeiten, und hält den Kontakt zum Deutschen Akademischen Austauschdienst (DAAD). Das Thema Auslandsfamulatur und die damit verbundenen Erfahrungen sind ein wichtiges Anliegen für den Freien Verband. Der ZAD strebt an, die Studenten über die reine Vermittlung von Famulaturen und Reisekostenzuschüssen hinaus zu unterstützen. Weitere Informationen sind

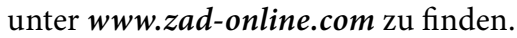

\section{Zum Tode von Dr. Richard V. Tucker}

\section{Perfektionist der Goldrestauration}

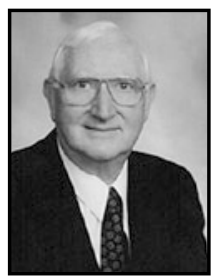

Sein Name steht für Zahnmedizin auf höchstem Niveau: Dr. Richard V. Tucker. Im Alter von fast 94 Jahren ist der US-Amerikaner, den viele Zahnärzte aus Fortbildungen zur sogenannten Tucker-Methode kennen, gestorben. In seiner fünfzigjährigen zahnärztlichen Tätigkeit entwickelte er seine weltweit anerkannte und reproduzierbare Methode zur Perfektionierung von Goldrestaurationen. Tucker wurde 1922 im US-amerikanischen Bundesstaat Idaho geboren und machte seinen Abschluss in Zahnmedizin an der Universität von Washington in Seattle kurz nach dem Zweiten Weltkrieg. Im Lau- fe der Jahre übernahm Tucker die hohen Qualitätsstandards der Goldfoil-Technik und Goldgusstechnik seines Mentors Dr. George Ellsperman und entwickelte sie weiter.

Obwohl schon in qualitätsorientierten Zahnärztegruppen in den USA bekannt, blieb die „Tucker-Technik“ in Europa lange Zeit ein Geheimtipp für wenige Eingeweihte. Heute existieren weltweit 73 Gruppen, deren 550 Mitglieder die Tuckertechnik studieren und in ihren Praxen anwenden. Tucker war bis ins hohe Alter ein international anerkannter und gefragter Referent und Lehrer und bildete viele junge Kollegen aus für eine hochwertige, dauerhafte restaurative Zahnmedizin. 